\title{
Extracellular vesicles in kidneys and their clinical potential in renal diseases
}

\author{
Sul A Lee ${ }^{1,2}$, Chulhee Choi $^{3,4}$, Tae-Hyun Yoo ${ }^{1}$ \\ ${ }^{1}$ Department of Internal Medicine and Institute of Kidney Disease Research, Yonsei University College of Medicine, Seoul, Republic of Korea \\ ${ }^{2}$ Department of Internal Medicine, MetroWest Medical Center, Framingham, MA, USA \\ ${ }^{3}$ ILIAS Biologics Inc., Daejeon, Republic of Korea \\ ${ }^{4}$ Department of Bio and Brain Engineering, KAIST, Daejeon, Republic of Korea
}

Extracellular vesicles (EVs), such as exosomes and microvesicles, are cell-derived lipid bilayer membrane particles, which deliver information from host cells to recipient cells. EVs are involved in various biological processes including the modulation of the immune response, cell-to-cell communications, thrombosis, and tissue regeneration. Different types of kidney cells are known to release EVs under physiologic as well as pathologic conditions, and recent studies have found that EVs have a pathophysiologic role in different renal diseases. Given the recent advancement in EV isolation and analysis techniques, many studies have shown the diagnostic and therapeutic potential of EVs in various renal diseases, such as acute kidney injury, polycystic kidney disease, chronic kidney disease, kidney transplantation, and renal cell carcinoma. This review updates recent clinical and experimental findings on the role of EVs in renal diseases and highlights the potential clinical applicability of EVs as novel diagnostics and therapeutics.

Keywords: Biomarkers, Exosomes, Extracellular vesicles, Immunity, Kidney diseases, Microvesicles

\section{Introduction}

Extracellular vesicles (EVs) refer to all endogenously produced membrane-bound vesicles that are released from cells into the extracellular space [1]. EVs contain various molecules, such as DNA, messenger RNA (mRNA), proteins, lipids, and microRNAs (miRNAs). Different subtypes of EVs have been described including exosomes, microvesicles or microparticles, apoptotic bodies, ectosomes, and oncosomes based on their origin, size, contents, and biogenesis (Table 1). In the narrow sense, EVs usually refer to exosomes or microvesicles. In this review, we will use the compre-

Received: November 3, 2020; Revised: January 12, 2021; Accepted: January 26, 2021

Editor: Sang Heon Song, Pusan National University, Busan, Republic of Korea

Correspondence: Tae-Hyun Yoo

Department of Internal Medicine and Institute of Kidney Disease Research, Yonsei University College of Medicine, 50-1 Yonsei-ro, Seodaemun-gu, Seoul 03722, Republic of Korea. E-mail: yoosy0316@yuhs.ac

ORCID: https://orcid.org/0000-0002-9183-4507

Chulhee Choi

ILIAS Biologics Inc., 40-20 Techno 6-ro, Yuseong-gu, Daejeon 34014, Republic of Korea. E-mail: cchoi@iliasbio.com

ORCID: https://orcid.org/0000-0001-5542-9136

Chulhee Choi and Tae-Hyun Yoo contributed equally to this manuscript.

Copyright (C 2021 by The Korean Society of Nephrology

(a) This is an Open Access article distributed under the terms of the Creative Commons Attribution Non-Commercial and No Derivatives License (http:// creativecommons.org/licenses/by-nc-nd/4.0/) which permits unrestricted non-commercial use, distribution of the material without any modifications, and reproduction in any medium, provided the original works properly cited. 
Table 1. Classification of extracellular vesicles

\begin{tabular}{|c|c|c|c|}
\hline Variable & Exosome & Microvesicle & Apoptotic body \\
\hline Formation & Endosomal pathway, exocytosis & $\begin{array}{l}\text { Outward blebbing of the plasma } \\
\text { membrane }\end{array}$ & Cell shrinkage and fragmentation \\
\hline Size & $30-100 \mathrm{~nm}$ & $100-1,000 \mathrm{~nm}$ & $1-5 \mu \mathrm{m}$ \\
\hline Content & $\begin{array}{l}\text { Proteins, lipids, mRNA, miRNA, and } \\
\text { cytosol }\end{array}$ & $\begin{array}{l}\text { Proteins, lipids, mRNA, miRNA, and } \\
\text { cytosol }\end{array}$ & $\begin{array}{l}\text { Proteins, lipids, nuclear fractions, } \\
\text { DNA, rRNA, organelles, and cytosol }\end{array}$ \\
\hline Main protein markers & $\begin{array}{l}\text { Tetraspanins (CD63, CD9), Alix, and } \\
\text { TSG101 }\end{array}$ & \multicolumn{2}{|c|}{ Integrins, selectins, and CD40 ligand Histones } \\
\hline $\begin{array}{l}\text { Appearance by electron } \\
\text { microscopy }\end{array}$ & Cup shape & Irregular shape & Heterogenous \\
\hline
\end{tabular}

mRNA, messenger RNA; miRNA, microRNA; rRNA, ribosomal RNA; TSG101, tumor susceptibility gene 101.

hensive term 'EVs' to focus on exosomes and microvesicles because of overlapping characteristics among the different subtypes. EVs were initially discovered over 30 years ago, but it was thought that their physiological role was limited to the excretion of intracellular or membrane components of cells. However, recent advancements have shown that EVs are involved in multiple biological processes such as immune modulation, hemostasis, and tissue proliferation/ regeneration, which can affect the development and regeneration of organs [1-3]. EVs are also widely found in pathologic conditions. Their presence in any body fluid expands the diagnostic role of EVs as biomarkers in various types of diseases. EVs also contribute to disease progression by affecting the level of inflammation, thrombosis, and tumorigenesis $[4,5]$. On the other hand, EVs have received a lot of medical attention as a potential therapeutic vehicle based on their capacity of shuttling proteins and genetic materials. In this review, we will focus on the role of EVs in renal physiology and disease processes as well as their potential applicability as diagnostics and therapeutics in multiple renal diseases.

\section{Physiologic role of extracellular vesicles in kidneys}

In physiologic conditions, the major biological functions of EVs are to get rid of unwanted substances from host cells. EVs can also transfer important biological information to their recipient cells by delivering genetic material, proteins, lipids, and receptors. The conveyance of RNAs or miRNAs can have a substantial effect on the recipient cells by reprogramming their genetic characteristics. Below, we will discuss in detail the biological function of EVs in renal physiology (Fig. 1A [6]).

\section{Homeostasis and cell survival}

One of the most important biological roles of EVs is in the removal of intracellular toxic materials to extracellular spaces. As cells go through multiple biological processes, they build up damaged organelles and cellular waste, which can induce cellular stress leading to cell death or inflammation. Eukaryotic cells have developed a self-defense mechanism for the removal of intracellular waste-the secretion of EVs into the extracellular space [7]. In this way, cells can effectively eliminate potentially harmful chromosomal DNA fragments through exosomes. The inhibition of this process can induce an innate immune response, DNA damage, and apoptosis in normal human cells. Kidney cells are relatively susceptible to biological stress. Under hypoxic conditions, renal tubular cells proactively augment the amount of exosome secretion and alter the composition of exosomes to meet specific biological needs [8]. EV uptake into recipient cells is also a very important biological process in intercellular communication. The most common mechanism of cellular uptake is endocytosis whereby the EVs are engulfed by recipient cells [9]. The balance between EV release and uptake depends on the physiologic condition of the parent/ recipient cells, the type of parent/recipient cells, and the recognition of ligands/ receptors on the EVs and recipient cells [10].

\section{Inflammation/immunomodulation}

EVs are a miniature version of their parent cells, so their immunologic function significantly depends on their origin and the microenvironment their parent cells are exposed to. For example, EVs from dendritic cells can function as 

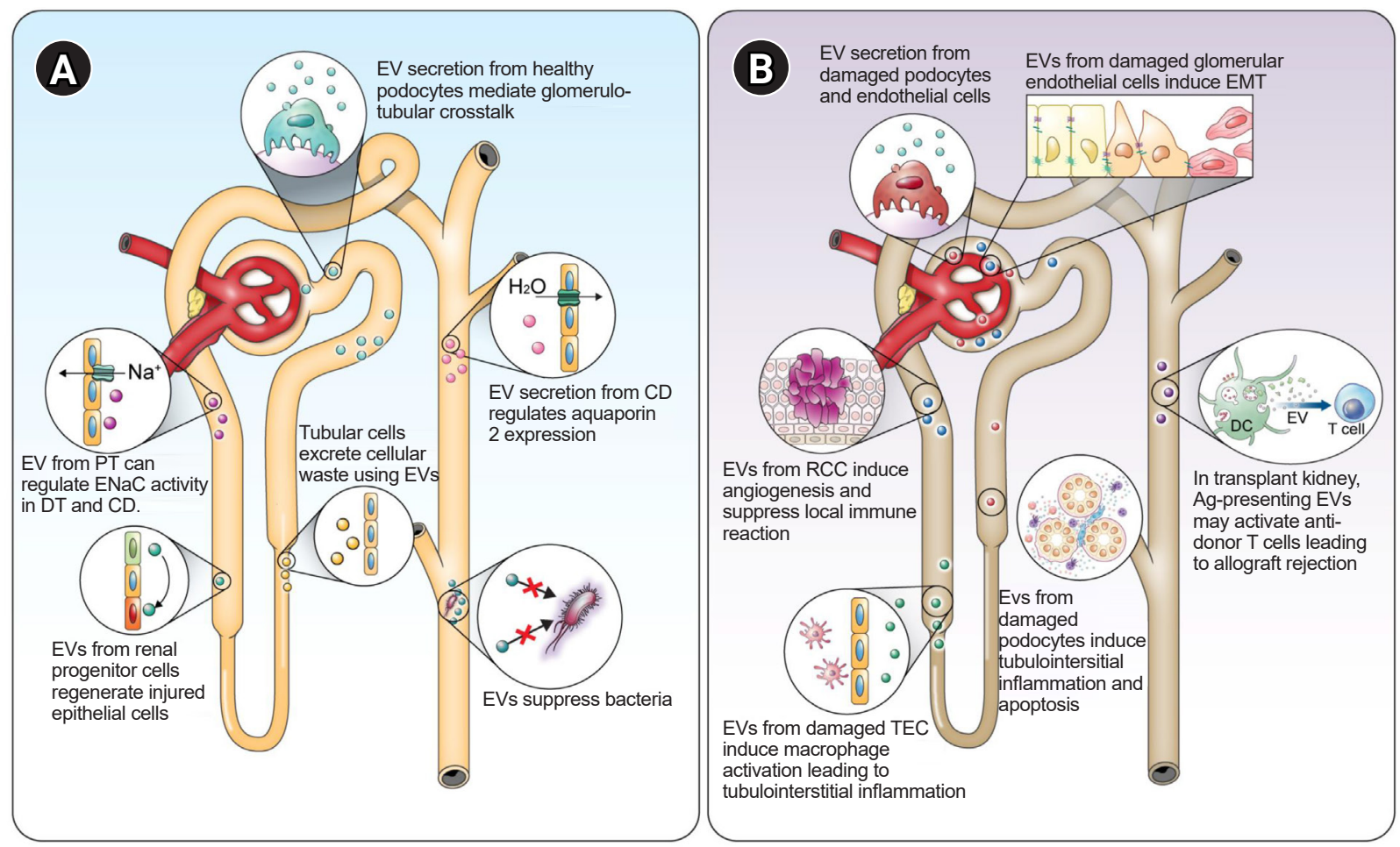

Figure 1. Role of extracellular vesicles (EVs) in normal kidneys and renal disease. (A) EVs mediate cell-to-cell communications, modulating cellular homeostasis, electrolyte/water balance, tubular regeneration, and inflammatory reactions in normal kidneys. (B) EVs affect disease progression by amplifying inflammation, inducing tubulointerstitial fibrosis or glomerular epithelial-mesenchymal transition (EMT). EVs may also be involved in the pathogenesis of renal cell carcinoma (RCC) and renal allograft rejection.

Ag, antigen; CD, collecting duct; DC, dendritic cell; DT, distal tubule; ENaC; epithelial sodium channel; PT, proximal tubule; TEC, tubular epithelial cells.

Modified from the article of Kwon et al. (Korean J Intern Med 2019;34:470-479) [6] under the Creative Commons License.

antigen-presenting vesicles through the direct presentation of peptide-major histocompatibility complex (MHC) complexes to T cells [11]. These peptide-MHC complexes can be transferred to other recipient cells, indirectly presenting antigens, and subsequently leading to immune cell stimulation. On the other hand, EVs secreted by tumor cells can mediate an immuno-suppressive response by switching monocytes and $\mathrm{T}$ cells to tolerogenic subtypes, inhibiting the differentiation of monocytes, or inducing the apoptosis of bystander $\mathrm{T}$ cells, so-called activation-induced cell death [12]. The physiological state of the parent cells significantly affects the content of the EVs. For example, exosomes derived from hypoxic tumor cells could induce higher levels of differentiation and the activation of myeloid-derived suppressor cells compared to exosomes derived from cells under normoxic conditions [13].

\section{Antimicrobial effect}

The urinary tract system is constantly exposed to microorganisms from the exterior environment, putting it at high risk of urinary tract infection. However, most of the urinary tract, except the urethra, generally remains sterile. This resistance to infection is mediated by several factors. Traditionally, anatomical barriers, such as the glycoprotein plaque and a layer of hydrated mucus, as well as immunologic barriers from various resident immune cells and the epithelial cell lining, were considered to be major host defense mechanisms [14]. Recently, EVs were found to serve a significant role against infection within the urinary tract. A proteomic study showed that enriched innate immune proteins including calprotectin and lysozyme $\mathrm{C}$ in urinary EVs could mediate an antimicrobial effect [15]. Even though autophagy is a well-developed cellular defense mechanism to clear foreign pathogens, some 
bacteria have evolved defense mechanisms to overcome autophagy by neutralizing lysosomal $\mathrm{pH}$. A study by Miao et al. [16] shows that bladder epithelial cells can overcome this phenomenon through exosomes-by expulsing exosome-encased bacteria from the intracellular space.

\section{Kidney regeneration/repair}

Mesenchymal stem cells (MSCs) are well-known for their regenerative and reparative potential in different organs including the kidneys [17]. However, the reparative potential of MSCs is mediated through EVs rather than the differentiation potential of the MSCs themselves. MSC-derived EVs contain different types of antioxidants and growth factors to stimulate the differentiation of resident progenitor cells. Delivery of genetic materials like DNA, miRNAs, or mRNAs through EVs can also introduce the reparative potential of parental MSCs to recipient cells. In a study by Ranghino et al. [18], glomerular MSC-derived EVs mediated tubular epithelial cell regeneration and alleviated ischemic acute kidney injury (AKI) by transferring mRNAs and miRNAs with potential proregenerative effects.

\section{Hemostasis and platelet aggregation}

An ample number of publications have reported the coagulant properties of plasma EVs in humans [19,20]. EVs originating from platelets, endothelial cells, or leukocytes were found to be involved in the course of hemostasis. These EVs can initiate the coagulation process by exposing phosphatidylserine (PS) on their surfaces to allow binding sites for coagulation factors [21]. Another mechanism is through the expression of tissue factors on their surfaces, initiating the extrinsic pathway of coagulation and leading to thrombin burst and platelet clot formation [22]. A study by Yu et al. [23] showed that patients with diabetic kidney disease had a significantly higher level of PS-expressing microvesicles compared to control groups. They also found that higher levels of PS-positive microvesicles were associated with hypercoagulable status as well as worse renal function. A similar finding was shown in patients with immunoglobulin A nephropathy [24].

Recently, several studies have revisited the procoagulation property of EVs because of technical improvements in blood collection, plasma preparation, and EV isolation [25]. Interestingly, plasma EVs isolated from healthy individuals pro- mote fibrinolysis more dominantly than coagulation. This discrepancy suggests an ambivalent role of EVs in hemostasis processes, which depends on the microenvironment. In addition, proper EV sample preparation is important for a better understanding of their role in the human body.

\section{Vessel integrity and revascularization}

Endothelial cell-derived EVs help to maintain vessel integrity and promote vascular endothelial cell survival by removing cell-death signals and releasing excess complements from vascular endothelial cells [26]. In damaged vessels, platelet-derived EVs induce the adhesion of endothelial cells to the extracellular matrix, which helps the regeneration of endothelium and attenuates vascular permeability [27].

Tumor-derived EVs are well-known for their angiogenetic properties, but EVs can also induce angiogenesis under physiologic conditions. Endothelial cell-derived EVs modulate angiogenesis by promoting endothelial cell invasion and capillary-like structure formation [28]. Recently, the transfer of certain miRNAs, including miR-126 or miR-124, using endothelial cell-derived EVs and recipient endothelial cells has been found to have a significant role in angiogenesis [29,30]. MSC-induced EVs also induce angiogenesis by transferring proteins like phosphorylated signal transducer and activator of transcription-3 or nuclear factor-kB pathway-associated proteins, leading to the transcription of proangiogenic proteins [31,32]. MSC-derived EVs can also transfer several growth factors including epidermal growth factor and vascular endothelial growth factor to endothelial cells promoting angiogenesis. More detailed information about the angiogenetic mechanism of EVs from multiple cellular origins can be found in a review article by Todorova et al. [2] .

\section{Electrolyte and water balance}

Sodium/water reabsorption is one of the major functions of renal tubular cells. Two-thirds of filtrated sodium is reabsorbed in proximal tubular epithelial cells and fine-tuning for sodium reabsorption occurs in the distal tubule and collecting ducts. Therefore, interactive communication between proximal and distal/collecting tubular cells are important for proper maintenance of electrolyte balance and volume control. A study by Jella et al. [33] showed that exosomes from proximal tubular cells can regulate epithelial sodium channel 
activity in the distal tubule and collecting ducts through the exosomal delivery of glyceraldehyde 3-phosphate dehydrogenase. Upon exposure to vasopressin, cortical collecting duct tubular cells increase the production of aquaporin water channels within themselves as well as within their exosomes [34]. These exosomes could transfer functional aquaporin channels to other tubular cells, increasing their water transport capacity. By mediating inter and intracellular communication of renal epithelial cells among the different nephron segments, exosomes provide a significant role in electrolyte and fluid balance in the human body.

\section{Extracellular vesicles as mediators of kidney disease}

In pathologic conditions, the production of EVs significantly increases, contributing to the initiation and propagation of disease through immunomodulation, thrombogenesis, and oncogenesis. The detailed role of renal EVs in different disease courses are discussed below (Fig. 1B).

\section{Glomerular disease}

In advanced glomerular disease, tubular damage and fibrosis are the leading mechanisms of renal dysfunction. A recent study by Jeon et al. [35] showed that miRNAs in EVs released by injured podocytes can induce tubular epithelial cell apoptosis, suggesting possible glomerulotubular crosstalk. This study also showed that the miRNA profile in EVs from injured podocytes are different from those of non-injured podocytes, and miRNA mimics of injured podocytes could reproduce a proapoptotic effect on renal tubular epithelial cells. In diabetic nephropathy, exposure to high glucose levels can induce EV production from podocytes. Tubular epithelial cells uptake these podocyte-derived EVs and generate tubular fibrosis through p38 phosphorylation [36].

\section{Acute kidney injury}

A recent miRNA sequencing study showed that urinary exosomal miRNAs have characteristic profiles and functional properties that depend on the AKI stage [37]. Several exo-miRNAs (miR-16, miR-24, and miR-200c) were increased during the early injury state, and the expression of their target mRNAs was significantly affected in the renal medulla. During the early recovery phase, exo-miRNAs that share Zeb1/2 as a common target were significantly upregulated, implying their role in transforming growth factor (TGF)- $\beta 1$-associated renal fibrosis. In ischemia-reperfusion injury, hypoxic damage induces the increased production of EVs in tubular epithelial cells that contains TGF- $\beta 1$ mRNA [8]. Exosomal delivery of these TGF- $\beta 1$ mRNAs into fibroblasts ultimately leads to fibroblast activation and proliferation. On the other hand, Ranghino et al. [18] recently showed that MSCs within the glomeruli can contribute to postischemic renal recovery primarily through the release of EVs.

In sepsis-related AKI, platelet-derived and neutrophil-derived EVs demonstrate proinflammatory and procoagulant effects, which can prevent the growth of bacteria and its dissemination [38]. However, these EVs can systematically induce oxidative stress and disseminate inflammatory responses, leading to poorer clinical outcomes.

\section{Tubulointerstitial inflammation}

Tubulointerstitial inflammation is a common characteristic of both acute and chronic kidney disease. Lv et al. [39] found a significant increase in tubular epithelial cell-derived exosomal miRNA-19b-3p in both a lipopolysaccharide-induced AKI model and an adriamycin-induced chronic proteinuric kidney disease model. Tubular epithelial cell-derived exosomal miRNA-19b-3p promoted M1 macrophage activation and tubulointerstitial inflammation. This group also showed that exosomal delivery of CCL2 mRNA from tubular epithelial cells to macrophages could mediate albumin-induced tubulointerstitial inflammation [40]. Hypoxia is another well-known stimulator of tubulointerstitial inflammation in the kidney. Hypoxic stimulation induces the expression of hypoxia-inducible factor- $1 \alpha$ in tubular epithelial cells. This stimulus leads to exosomal miRNA-23a transfer from tubular epithelial cells to macrophages, which triggers macrophage activation and promotes tubulointerstitial inflammation [41]. Thus, modulation of macrophage activation by blocking exosomal miRNA or mRNA delivery from tubular epithelial cells can be a novel therapeutic approach.

\section{Fibrosis}

Fibrosis in glomeruli and tubules is an ultimate pathologic 
process of chronic kidney diseases and end-stage renal disease, and the pathophysiological role of EVs in fibrosis has been explored in several studies $[5,8,42,43]$. Various stimuli on renal cells, such as hypoxia, oxidative stress, or high glucose, can lead to EV secretion or changes in the EV composition $[8,42]$. EVs secreted by damaged kidney cells can be transferred to other normal kidney cells, changing their phenotype and activating fibroblasts. In hypoxic conditions, damaged tubular epithelial cells transfer TGF- $\beta 1$ mRNA to fibroblasts through exosome secretion, ultimately leading to the proliferation/activation of fibroblasts and progression of renal tubular fibrosis [8]. An in vitro study by Wu et al. [42] showed that EVs secreted from high glucose-treated glomerular endothelial cells can trigger podocyte epithelial-mesenchymal transition by transferring TGF- $\beta 1$ mRNA into glomerular mesangial cells. This process induces cellular proliferation and overproduction of extracellular matrix within glomeruli, which is a key pathological mechanism of diabetic nephropathy. Several miRNAs in EVs, such as miR-21, miR-192, and miR-34a, also affect the biological processes of tubular fibrosis $[43,44]$ and glomerular fibrosis [45], respectively.

\section{Nephrolithiasis}

Given the significant role of EVs as mediators of various renal diseases, several studies have speculated that EVs have a role in stone formation. For instance, an in vitro study by He et al. [46] showed that exposure of renal tubular cells to different concentrations of oxalates could affect the size and composition of the exosomes from tubular cells. This result suggests that the biological role of exosomes may depend on the level of oxalate exposure in a given microenvironment. Other in vitro studies by Singhto et al. $[47,48]$ showed that exosomes derived from human macrophages exposed to calcium oxalate crystals could induce the migration/ activation of monocytes/T-cell/neutrophils and enhance the production of proinflammatory cytokine interleukin-8. These exosomes had a greater capacity to bind calcium oxalate crystals and subsequently enhanced crystal invasion through the extracellular matrix, suggesting the possible role of macrophage-derived exosomes in the crystal invasion of the renal interstitium.

\section{Renal cancer}

EVs mediate intercellular communication between tumor cells. EVs from cancer cells contain proangiogenic mRNAs and various miRNAs with tumor invasive properties [49]. Clear renal cell carcinoma-derived EVs can negatively affect adjacent immune cells by the activation of the TGF- $\beta / S M A D$ signaling pathway [50]. EVs are also known to mediate chemoresistance in renal cancer cells through the delivery of long noncoding/competing endogenous RNA, ultimately leading to higher expression of prometastatic receptors [51]. Further information about the role of EVs in urological malignancies is well described in a review article by Linxweiler and Junker [4].

\section{Extracellular vesicles as biomarkers in various kidney diseases}

Most of the EV studies in kidney diseases have been focused on biomarker discoveries. EVs can be found in multiple types of biological fluids including serum, urine, breast milk, cerebrospinal fluid, saliva, ascites, and bronchoalveolar lavage fluid. Under physiologic conditions, almost all urinary EVs originate from kidney cells because circulating EVs do not generally go through the glomerular basement membrane. Therefore, the organ-specific origin of urinary EVs comprises the major advantage of using urinary EVs as biomarkers in kidney diseases. Also, urinary EVs can be obtained noninvasively and repeatedly, providing important information regarding diagnosis, prognosis, and response to treatment.

Even though circulating EVs can originate from different organs, serum, and plasma, they can still provide significant information about the extent and prognosis of renal disease because the clinical outcome is determined not only from the kidney damage itself but also from the dysfunction of distant organs including the lungs, heart, brain, liver, and immune system [52]. Easier clinical accessibility is also a huge benefit of using circulating EVs as biomarkers in kidney disease.

Recent advancements in 'omic' studies have expedited the discovery of novel EV biomarkers enabling the detection of pathologic processes even earlier than traditional biomarkers. Salih et al. [53] compared urinary EVs from autosomal dominant polycystic kidney disease (ADPKD) patients and 
healthy controls using quantitative proteomics. Among the proteins with higher abundancy, five proteins including complements and plakins were selected for further analysis based on their significance in pathway analysis. Especially, a higher level of complement in urinary EVs was found in younger ADPKD patients with preserved kidney function, raising its potential as a biomarker. Due to the vast amount of information from various studies and limited space, the role of EVs as biomarkers is summarized in Table 2 [53-78].

\section{Extracellular vesicles as therapeutics in various kidney diseases}

\section{Extracellular vesicles as a cargo of therapeutic material}

The role of EVs as biocarriers has received a lot of attention

Table 2. Extracellular vesicles as biomarkers in renal disorders

\begin{tabular}{|c|c|c|c|}
\hline Disease & Source/method & Main finding & Reference \\
\hline \multirow[t]{2}{*}{$\mathrm{AKI}$} & Human urine/Western blotting & $\begin{array}{l}\text { Increased level of } \mathrm{Na}^{+} / \mathrm{H}^{+} \text {exchanger type- } 3 \text { in urinary exosome was } \\
\text { found in patients with acute tubular necrosis but not in other etiologies } \\
\text { of AKI. }\end{array}$ & [54] \\
\hline & $\begin{array}{l}\text { Human or rat urine/Western } \\
\text { blotting }\end{array}$ & Increased urinary exosomal ATF3 in AKI and WT-1 in podocyte injury. & [56] \\
\hline \multirow[t]{2}{*}{ DN } & Human urine/RT-qPCR & $\begin{array}{l}\text { In DN patients, urinary exosomal let- } 7 c-5 p \text { was upregulated, and miR- } \\
29 c-5 p \text { and miR-15b-5p were down-regulated. These three miRNAs } \\
\text { could predict the development of DN. }\end{array}$ & [58] \\
\hline & Human urine/RT-qPCR & $\begin{array}{l}\text { Increased level of uromodulin mRNA in urinary EVs derived from } \\
\text { patients with type } 2 \mathrm{DM} \text { nephropathy compared to type } 2 \mathrm{DM} \text { without } \\
\text { nephropathy. Urinary EV uromodulin mRNA level correlates with renal } \\
\text { function decline in type } 2 \text { DM patients. }\end{array}$ & [59] \\
\hline \multirow[t]{3}{*}{ IgA nephropathy } & Human urine/RT-qPCR & $\begin{array}{l}\text { Total amount of urinary exosomes and exosomal CCL2 MRNA are } \\
\text { upregulated in IgA nephropathy patients, which reflects active renal } \\
\text { histologic injury and renal function deterioration. }\end{array}$ & [61] \\
\hline & Human serum/RT-qPCR & $\begin{array}{l}\text { IgA nephropathy patients with lower serum miR-182 had more severe } \\
\text { tubular atrophy, interstitial inflammation, and fibrotic tendency; IgA } \\
\text { nephropathy patients with higher serum miR-192 had a slower disease } \\
\text { progression. }\end{array}$ & [62] \\
\hline & $\begin{array}{l}\text { Human plasma/RNA- } \\
\text { sequencing }\end{array}$ & $\begin{array}{l}\text { Exosomal IncRNA-G21551 was significantly down-regulated in IgA } \\
\text { nephropathy patients compared to healthy first-degree relatives. }\end{array}$ & [63] \\
\hline Nephrotic syndrome & Human urine/RT-qPCR & $\begin{array}{l}\text { Urinary exosomal miR-193a is significantly increased in primary FSGS } \\
\text { patients compared to those in MCD patients. }\end{array}$ & [64] \\
\hline & $\begin{array}{l}\text { Human urine/integrated kidney } \\
\text { exosome analysis }\end{array}$ & $\begin{array}{l}\text { High level of CD3 positive urinary EVs in patients with acute cellular } \\
\text { rejection. }\end{array}$ & [68] \\
\hline & Human urine/proteomics & $\begin{array}{l}\text { Higher levels of tetraspanin-1 and hemopexin protein are found in } \\
\text { urinary exosomes derived from patients with T cell-mediated rejection. }\end{array}$ & [69] \\
\hline
\end{tabular}


Table 2. Continued

\begin{tabular}{|c|c|c|c|}
\hline Disease & Source/method & Main finding & Reference \\
\hline \multirow[t]{3}{*}{ PKD } & Human urine/proteomics & $\begin{array}{l}\text { Higher levels of complement C3 and C9 are found in urinary EVs derived } \\
\text { from ADPKD patients. }\end{array}$ & [53] \\
\hline & & $\begin{array}{l}\text { Increased levels of villin-1, periplakin, and envoplakin are found in } \\
\text { urinary EVs from patients with advanced ADPKD. }\end{array}$ & \\
\hline & $\begin{array}{l}\text { Human and rat urine/Western } \\
\text { blotting }\end{array}$ & Increased urinary exosomal AGS3 expression in PKD patients/rats. & [70] \\
\hline \multirow[t]{3}{*}{ LN } & Human urine/RT-PCR & $\begin{array}{l}\text { Urinary exosomal miR-29C correlates with the degree of renal chronicity } \\
\text { but not with renal function in patients with LN. }\end{array}$ & [71] \\
\hline & Human urine/RT-PCR & $\begin{array}{l}\text { Decreased level of urinary exosomal let-7a and miR-21 in patients with } \\
\text { active LN compared to inactive LN. }\end{array}$ & [72] \\
\hline & $\begin{array}{l}\text { Human urine/miRNA } \\
\text { sequencing }\end{array}$ & $\begin{array}{l}\text { Urinary exosomes from LN patients complicated with cellular crescent } \\
\text { have a unique miRNA expression profile including miR-3135b, miR- } \\
654-5 p \text {, and miR-146a-5p. }\end{array}$ & [73] \\
\hline $\begin{array}{l}\text { Renal fibrosis and } \\
\text { CKD model }\end{array}$ & Human urine/RT-qPCR & $\begin{array}{l}\text { Down-regulation of urinary exosomal miR-29c level with worsening renal } \\
\text { function and progression of tubulointerstitial fibrosis in CKD patients. }\end{array}$ & {$[74]$} \\
\hline \multirow[t]{2}{*}{ RCC } & Human plasma/RT-qPCR & $\begin{array}{l}\text { Increased hsa-miR-301a-3p and decreased hsa-miR-1293 levels } \\
\text { were found in plasma EVs in patients with metastatic clear cell RCC } \\
\text { compared to those with localized disease. }\end{array}$ & {$[77]$} \\
\hline & Human plasma/RT-qPCR & $\begin{array}{l}\text { Higher levels of plasma EV-derived TIMP-1 mRNA were found in patients } \\
\text { with metastatic RCC or huge tumor burden. Also, patients with a high } \\
\text { level of EV-derived TIMP-1 mRNA had poorer survival compared to } \\
\text { those with a low level. }\end{array}$ & [78] \\
\hline
\end{tabular}

ADPKD, autosomal dominant polycystic kidney disease; AGS3, activator of G-protein signaling 3; AKI, acute kidney injury; ATF3, activating transcription factor 3; CKD, chronic kidney disease; DN, diabetic nephropathy; DM, diabetes mellitus; EV, extracellular vesicle; FSGS, focal segmental glomerulosclerosis; IgA, immunoglobulin A; LN, lupus nephritis; IncRNA, long noncoding RNA; MCD, minimal change disease; miRNA and miR, microRNA; mRNA, messenger RNA; NGAL, neutrophil gelatinase-associated lipocalin; PKD, polycystic kidney disease; RCC, renal cell carcinoma; RT-qPCR, reverse transcriptionquantitative polymerase chain reaction; RT-PCR, reverse transcription-polymerase chain reaction; SH2D1B, SH2 domain-containing protein 1B; TIMP, tissue inhibitor of metalloproteinase; TNF-a, tumor necrosis factor alpha; WT-1, Wilms tumor 1.

recently. EVs carry several biological benefits as a vector over other methods, including stability, small size, and low immunogenicity. Specific cell surface molecules on EVs increase their targeting capacity with fewer off-target effects. Nanosized EVs with certain surface molecules can even penetrate natural barriers such as the blood-brain barrier [79]. Also, EVs use the native mechanisms of recipient cells in the course of internalization and intracellular trafficking [80]. Delivery of biomolecular cargos such as mRNA, miRNA, and proteins can modify the genetic profile and biological response of recipient cells, leading to the modulation of disease processes [81].

EV loading of traditional drugs with anti-inflammatory properties has been studied for therapeutic applicability $[82,83]$. Dexamethasone, for example, is widely used to suppress inflammation in various renal diseases, but its clinical use is limited due to significant adverse effects. By incubat- ing macrophages with dexamethasone, macrophage-derived dexamethasone containing EVs were successfully produced and delivered to the inflamed kidneys of mice, which showed significant suppression of renal inflammation and fibrosis [82]. Notably, dexamethasone containing EVs substantially decreased the adverse effects from systemic steroid treatment such as hyperglycemia and suppression of the hypothalamic-pituitary-adrenal axis.

During the past decade, there has been an explosive advancement in $\mathrm{EV}$ research. Interdisciplinary cooperation between medicine and biomedical engineering has harnessed EVs as potent drug delivery tools through the application of nanotechnology. Several studies have successfully loaded genetic material into EVs and shown effects on modifying disease courses. Yim et al. [84] recently developed a novel technique for the effective intracellular transfer of soluble proteins through exosomes by using optogenetic engineering. 
This technology was adopted in an experimental sepsis model, showing the protective effect of exosomal super-repressor inhibitor of kappa B ( $\mathrm{K} \mathrm{B}$ ) delivery in a murine septic AKI model [85]. Recently, our group has also demonstrated the beneficial effect of exosomal super-repressor I $\mathrm{B}$ delivery in an ischemic AKI model (under review).

\section{Extracellular vesicles for kidney repair}

Stem cell therapy has great benefits in multiple experimental kidney injury models, not through their differentiation potential but the paracrine effects of their EVs [17]. EVs secreted from MSCs or endothelial progenitor cells have been shown to play a significant role in the amelioration of tubular cell apoptosis/fibrosis and proliferation of tubular and endothelial cells in various AKI models [86,87]. This beneficial effect was also found in experimental chronic kidney disease models, ameliorating epithelial-to-mesenchymal transition and preventing inflammatory cell infiltration [88,89]. MSCs that were engineered to overexpress miRNA-let7c could selectively deliver miRNA-let7c to damaged kidney cells using exosomes, which led to protection from renal injury and decreased fibrosis in a unilateral ureteral obstruction model [90]. Nagaishi et al. [91] showed that MSC-derived renal trophic factors including exosomes could alleviate albuminuria and immune cell infiltration into the kidneys in a diabetic nephropathy model. MSC-derived exosomes showed an antiapoptotic effect and protected tight junction structure in tubular epithelial cells. Intravenous injections of urinary stem cell-derived exosomes could also reduce urinary microalbumin excretion and prevent podocyte/tubular epithelial cell apoptosis in diabetic rats [92].

Apart from MSCs, EVs from other origins have shown a renoprotective effect in animal kidney injury models. Exosomes derived from sera of animals after remote ischemic preconditioning could attenuate sepsis-induced renal damage, and this was mediated by the upregulation of exosomal miRNA-21 [93]. Dominguez et al. [94] showed that intravenous delivery of EVs from rat renal tubular cells could markedly reduce renal damage in recipients after ischemic insult. This group also showed that human renal exosomes can prevent ischemic renal injury in nude rats [95].

However, the clinical evidence of the renoprotective effect of EV therapy in humans is relatively scarce. Several problems limit the therapeutic application of EVs in humans; the low yield of EVs from cultured cells and premature EV isolation/ purification with a risk of contamination from other molecules with similar density $[96,97]$. Although several studies have shown promising advances in EV isolation and purification, which is reviewed elsewhere [98], further efforts are needed to improve isolation efficiency and purity. Also, large-scale production is required for the therapeutic application of EVs in humans. Yang et al. [99] recently used a cellular nanoporation method to produce large-scale exosomes containing therapeutic mRNAs and targeting peptides. By using this strategy, they could produce a significantly higher number of exosomes and exosomal mRNA transcripts compared with bulk electroporation and other exosome production strategies. Lastly, advanced techniques in the fine modulation of EV content using transfection or cell engineering is needed to deliver specific transcripts or protein composition into target cells. This will lead to a better understanding of the biological role of certain EVs in specific renal disease models and will broaden the therapeutic applicability of EVs in various renal diseases.

\section{Extracellular vesicles as potential therapeutic targets}

As we have mentioned, EVs can mediate the propagation of renal damage in septic, inflammatory, or thrombogenic conditions. Therefore, temporarily blocking the release and uptake of EVs can possibly alleviate tissue damage. Various pharmacologic agents including antiplatelet agents, statins, calcium channel blockers, and abciximab were found to negatively affect the process of EV release and uptake, which is reviewed elsewhere [1]. However, whether blocking the release and uptake of EVs can directly affect the disease course has not been fully investigated. A study by Mossberg et al. [100] showed that $\mathrm{Cl}$-inhibitor can significantly reduce the release of chemotactic kinin B1-receptor-positive endothelial microvesicles, suggesting it has therapeutic potential in inflammatory diseases. Further studies are required to understand the clinical applicability of controlling EV interaction with recipient cells in systemic inflammatory conditions.

\section{Conclusions and perspectives}

EVs are involved in cell-to-cell communications within kidneys, mediating podocyte-tubulointerstitial/proximal-distal tubular/glomerulus-tubular crosstalk. EVs can either pro- 
vide physiological roles in renal repair and immunomodulation or mediate pathological processes inducing thrombosis and inflammation. Recent studies have significantly improved our understanding of the roles of EVs in kidney physiology and pathology. However, most of the results are limited to descriptive transcriptomic/proteomic analysis and lack consistency as well as reliability. Stringent validation through clinical trials and large-scale cohort studies is necessary before entering into clinical application. Further optimization of $\mathrm{EV}$ isolation techniques and meticulous manipulation of the genetic materials or protein compositions of EVs are also necessary for clinical consideration. These efforts will extend our knowledge on the role of EVs in the development/ progression of kidney diseases and will broaden the clinical applicability of EVs as novel diagnostics and therapeutics of renal diseases.

\section{Conflicts of interest}

Tae-Hyun Yoo is a Scientific Advisory Board member at ILIAS Biologics Inc. and the Editor-in-Chief of Kidney Research and Clinical Practice. Chulhee Choi is the founder and a shareholder of ILIAS Biologics Inc. The authors have no additional financial interests.

\section{Funding}

This work was supported by grants from the National Research Foundation of Korea (NRF-2017R1A2B4005720, NRF-2017R1A2B3002241, NRF-2018M3A9E2022820, NRF2019R1A2C2084535), which is funded by the Korean government (MSIP).

\section{Acknowledgments}

The authors also thank Medical Illustration \& Design, part of the Medical Research Support Services of Yonsei University College of Medicine, for all artistic support related to this work.

\section{Authors' contributions}

Conceptualization, Data curation, Investigation, Visualization: SAL

Formal analysis, Project administration: CC, THY
Writing-original draft: SAL

Writing-review \& editing: CC, THY

All authors read and approved the final manuscript.

\section{ORCID}

Sul A Lee, https://orcid.org/0000-0003-2121-721X

Chulhee Choi, https://orcid.org/0000-0001-5542-9136

Tae-Hyun Yoo, https://orcid.org/0000-0002-9183-4507

\section{References}

1. Karpman D, Ståhl AL, Arvidsson I. Extracellular vesicles in renal disease. Nat Rev Nephrol 2017;13:545-562.

2. Todorova D, Simoncini S, Lacroix R, Sabatier F, Dignat-George F. Extracellular vesicles in angiogenesis. Circ Res 2017;120:16581673.

3. Lipschutz JH. The role of the exocyst in renal ciliogenesis, cystogenesis, tubulogenesis, and development. Kidney Res Clin Pract 2019;38:260-266.

4. Linxweiler J, Junker K. Extracellular vesicles in urological malignancies: an update. Nat Rev Urol 2020;17:11-27.

5. Jing H, Tang S, Lin S, Liao M, Chen H, Zhou J. The role of extracellular vesicles in renal fibrosis. Cell Death Dis 2019;10:367.

6. Kwon SH. Extracellular vesicles in renal physiology and clinical applications for renal disease. Korean J Intern Med 2019;34:470479.

7. Takahashi A, Okada R, Nagao K, et al. Exosomes maintain cellular homeostasis by excreting harmful DNA from cells. Nat Commun 2017;8:15287.

8. Borges FT, Melo SA, Özdemir BC, et al. TGF- $\beta 1$-containing exosomes from injured epithelial cells activate fibroblasts to initiate tissue regenerative responses and fibrosis. J Am Soc Nephrol 2013;24:385-392

9. Yáñez-Mó M, Siljander PR, Andreu Z, et al. Biological properties of extracellular vesicles and their physiological functions. $J$ Extracell Vesicles 2015;4:27066.

10. Ståhl AL, Johansson K, Mossberg M, Kahn R, Karpman D. Exosomes and microvesicles in normal physiology, pathophysiology, and renal diseases. Pediatr Nephrol 2019;34:11-30.

11. Segura E, Nicco C, Lombard B, et al. ICAM-1 on exosomes from mature dendritic cells is critical for efficient naive T-cell priming. Blood 2005;106:216-223.

12. Clayton A, Mitchell JP, Court J, Mason MD, Tabi Z. Human tumor-derived exosomes selectively impair lymphocyte responses 
to interleukin-2. Cancer Res 2007;67:7458-7466.

13. Guo X, Qiu W, Liu Q, et al. Immunosuppressive effects of hypoxia-induced glioma exosomes through myeloid-derived suppressor cells via the miR-10a/Rora and miR-21/Pten pathways. Oncogene 2018;37:4239-4259.

14. Wu XR, Kong XP, Pellicer A, Kreibich G, Sun TT. Uroplakins in urothelial biology, function, and disease. Kidney Int 2009;75:11531165.

15. Hiemstra TF, Charles PD, Gracia T, et al. Human urinary exosomes as innate immune effectors. J Am Soc Nephrol 2014;25:2017-2027.

16. Miao Y, Li G, Zhang X, Xu H, Abraham SN. A TRP channel senses lysosome neutralization by pathogens to trigger their expulsion. Cell 2015;161:1306-1319.

17. Bochon B, Kozubska M, Surygała G, et al. Mesenchymal stem cells-potential applications in kidney diseases. Int J Mol Sci 2019; 20:2462.

18. Ranghino A, Bruno S, Bussolati B, et al. The effects of glomerular and tubular renal progenitors and derived extracellular vesicles on recovery from acute kidney injury. Stem Cell Res Ther 2017;8:24.

19. Gheldof D, Haguet H, Dogné JM, et al. Procoagulant activity of extracellular vesicles as a potential biomarker for risk of thrombosis and DIC in patients with acute leukaemia. J Thromb Thrombolysis 2017;43:224-232.

20. Ammollo CT, Semeraro F, Milella RA, Antonacci D, Semeraro N, Colucci M. Grape intake reduces thrombin generation and enhances plasma fibrinolysis. Potential role of circulating procoagulant microparticles. J Nutr Biochem 2017;50:66-73.

21. Skotland T, Hessvik NP, Sandvig K, Llorente A. Exosomal lipid composition and the role of ether lipids and phosphoinositides in exosome biology. J Lipid Res 2019;60:9-18.

22. Del Conde I, Shrimpton CN, Thiagarajan P, López JA. Tissue-factor-bearing microvesicles arise from lipid rafts and fuse with activated platelets to initiate coagulation. Blood 2005;106:16041611.

23. Yu M, Xie R, Zhang Y, et al. Phosphatidylserine on microparticles and associated cells contributes to the hypercoagulable state in diabetic kidney disease. Nephrol Dial Transplant 2018;33:21152127.

24. He Z, Zhang Y, Cao M, et al. Increased phosphatidylserine-exposing microparticles and their originating cells are associated with the coagulation process in patients with IgA nephropathy. Nephrol Dial Transplant 2016;31:747-759.

25. Berckmans RJ, Lacroix R, Hau CM, Sturk A, Nieuwland R. Extracellular vesicles and coagulation in blood from healthy humans revisited. J Extracell Vesicles 2019;8:1688936.
26. Abid Hussein MN, Böing AN, Sturk A, Hau CM, Nieuwland R. Inhibition of microparticle release triggers endothelial cell apoptosis and detachment. Thromb Haemost 2007;98:1096-1107.

27. Miyazawa B, Trivedi A, Togarrati PP, et al. Regulation of endothelial cell permeability by platelet-derived extracellular vesicles. $J$ Trauma Acute Care Surg 2019;86:931-942.

28. Taraboletti G, D'Ascenzo S, Borsotti P, Giavazzi R, Pavan A, Dolo V. Shedding of the matrix metalloproteinases MMP-2, MMP-9, and MT1-MMP as membrane vesicle-associated components by endothelial cells. Am J Pathol 2002;160:673-680.

29. Lombardo G, Dentelli P, Togliatto G, et al. Activated STAT5 trafficking via endothelial cell-derived extracellular vesicles controls IL-3 pro-angiogenic paracrine action. Sci Rep 2016;6:25689.

30. van Balkom BW, de Jong OG, Smits M, et al. Endothelial cells require miR-214 to secrete exosomes that suppress senescence and induce angiogenesis in human and mouse endothelial cells. Blood 2013;121:3997-4006.

31. Anderson JD, Johansson HJ, Graham CS, et al. Comprehensive proteomic analysis of mesenchymal stem cell exosomes reveals modulation of angiogenesis via nuclear factor-kappaB signaling. Stem Cells 2016;34:601-613.

32. Shabbir A, Cox A, Rodriguez-Menocal L, Salgado M, Van Badiavas E. Mesenchymal stem cell exosomes induce proliferation and migration of normal and chronic wound fibroblasts, and enhance angiogenesis in vitro. Stem Cells Dev 2015;24:1635-1647.

33. Jella KK, Yu L, Yue Q, Friedman D, Duke BJ, Alli AA. Exosomal GAPDH from proximal tubule cells regulate ENaC activity. PLoS One 2016;11:e165763.

34. Street JM, Birkhoff W, Menzies RI, Webb DJ, Bailey MA, Dear JW. Exosomal transmission of functional aquaporin 2 in kidney cortical collecting duct cells. J Physiol 2011;589:6119-6127.

35. Jeon JS, Kim E, Bae YU, et al. microRNA in extracellular vesicles released by damaged podocytes promote apoptosis of renal tubular epithelial cells. Cells 2020;9:1409.

36. Munkonda MN, Akbari S, Landry C, et al. Podocyte-derived microparticles promote proximal tubule fibrotic signaling via p38 MAPK and CD36. J Extracell Vesicles 2018;7:1432206.

37. Sonoda H, Lee BR, Park KH, et al. miRNA profiling of urinary exosomes to assess the progression of acute kidney injury. Sci Rep 2019;9:4692.

38. Mortaza S, Martinez MC, Baron-Menguy C, et al. Detrimental hemodynamic and inflammatory effects of microparticles originating from septic rats. Crit Care Med 2009;37:2045-2050.

39. Lv LL, Feng Y, Wu M, et al. Exosomal miRNA-19b-3p of tubular epithelial cells promotes M1 macrophage activation in kidney 
injury. Cell Death Differ 2020;27:210-226.

40. Lv LL, Feng Y, Wen Y, et al. Exosomal CCL2 from tubular epithelial cells is critical for albumin-induced tubulointerstitial inflammation. J Am Soc Nephrol 2018;29:919-935.

41. Li ZL, Lv LL, Tang TT, et al. HIF-1 $\alpha$ inducing exosomal microRNA-23a expression mediates the cross-talk between tubular epithelial cells and macrophages in tubulointerstitial inflammation. Kidney Int 2019;95:388-404.

42. Wu XM, Gao YB, Cui FQ, Zhang N. Exosomes from high glucose-treated glomerular endothelial cells activate mesangial cells to promote renal fibrosis. Biol Open 2016;5:484-491.

43. Zhou Y, Xiong M, Fang L, et al. miR-21-containing microvesicles from injured tubular epithelial cells promote tubular phenotype transition by targeting PTEN protein. Am J Pathol 2013;183:11831196.

44. Jia Y, Zheng Z, Guan M, et al. Exendin-4 ameliorates high glucose-induced fibrosis by inhibiting the secretion of miR-192 from injured renal tubular epithelial cells. Exp Mol Med 2018;50:1-13.

45. Zhang L, He S, Guo S, et al. Down-regulation of miR-34a alleviates mesangial proliferation in vitro and glomerular hypertrophy in early diabetic nephropathy mice by targeting GAS1. J Diabetes Complications 2014;28:259-264.

46. He Z, Guan X, Liu Y, et al. Alteration of exosomes secreted from renal tubular epithelial cells exposed to high-concentration oxalate. Oncotarget 2017;8:92635-92642.

47. Singhto N, Kanlaya R, Nilnumkhum A, Thongboonkerd V. Roles of macrophage exosomes in immune response to calcium oxalate monohydrate crystals. Front Immunol 2018;9:316.

48. Singhto N, Thongboonkerd V. Exosomes derived from calcium oxalate-exposed macrophages enhance IL-8 production from renal cells, neutrophil migration and crystal invasion through extracellular matrix. J Proteomics 2018;185:64-76.

49. Grange C, Tapparo M, Collino F, et al. Microvesicles released from human renal cancer stem cells stimulate angiogenesis and formation of lung premetastatic niche. Cancer Res 2011;71:53465356 .

50. Xia Y, Zhang Q, Zhen Q, et al. Negative regulation of tumor-infiltrating NK cell in clear cell renal cell carcinoma patients through the exosomal pathway. Oncotarget 2017;8:37783-37795.

51. Qu L, Ding J, Chen C, et al. Exosome-transmitted lncARSR promotes sunitinib resistance in renal cancer by acting as a competing endogenous RNA. Cancer Cell 2016;29:653-668.

52. Lee SA, Cozzi M, Bush EL, Rabb H. Distant organ dysfunction in acute kidney injury: a review. Am J Kidney Dis 2018;72:846-856.

53. Salih M, Demmers JA, Bezstarosti K, et al. Proteomics of urinary vesicles links plakins and complement to polycystic kidney disease. J Am Soc Nephrol 2016;27:3079-3092.

54. du Cheyron D, Daubin C, Poggioli J, et al. Urinary measurement of $\mathrm{Na}+\mathrm{H}+$ exchanger isoform 3 (NHE3) protein as new marker of tubule injury in critically ill patients with ARF. Am J Kidney Dis 2003;42:497-506.

55. Zhou H, Pisitkun T, Aponte A, et al. Exosomal Fetuin-A identified by proteomics: a novel urinary biomarker for detecting acute kidney injury. Kidney Int 2006;70:1847-1857.

56. Zhou H, Cheruvanky A, Hu X, et al. Urinary exosomal transcription factors, a new class of biomarkers for renal disease. Kidney Int 2008;74:613-621.

57. Panich T, Chancharoenthana W, Somparn P, Issara-Amphorn J, Hirankarn N, Leelahavanichkul A. Urinary exosomal activating transcriptional factor 3 as the early diagnostic biomarker for sepsis-induced acute kidney injury. BMC Nephrol 2017;18:10.

58. Li W, Yang S, Qiao R, Zhang J. Potential value of urinary exosome-derived let-7c-5p in the diagnosis and progression of type II diabetic nephropathy. Clin Lab 2018;64:709-718.

59. Yamamoto CM, Murakami T, Oakes ML, et al. Uromodulin mRNA from urinary extracellular vesicles correlate to kidney function decline in type 2 diabetes mellitus. Am J Nephrol 2018;47:283-291.

60. Prabu P, Rome S, Sathishkumar C, et al. MicroRNAs from urinary extracellular vesicles are non-invasive early biomarkers of diabetic nephropathy in type 2 diabetes patients with the 'Asian Indian phenotype'. Diabetes Metab 2019;45:276-285.

61. Feng Y, Lv LL, Wu WJ, et al. Urinary exosomes and exosomal CCL2 mRNA as biomarkers of active histologic injury in IgA nephropathy. Am J Pathol 2018;188:2542-2552.

62. Fan Q, Lu R, Zhu M, et al. Serum miR-192 is related to tubulointerstitial lesion and short-term disease progression in IgA nephropathy. Nephron 2019;142:195-207.

63. Guo N, Zhou Q, Huang X, et al. Identification of differentially expressed circulating exosomal lncRNAs in IgA nephropathy patients. BMC Immunol 2020;21:16.

64. Huang Z, Zhang Y, Zhou J, Zhang Y. Urinary exosomal miR193a can be a potential biomarker for the diagnosis of primary focal segmental glomerulosclerosis in children. Biomed Res Int 2017;2017:7298160.

65. Chen T, Wang C, Yu H, et al. Increased urinary exosomal microRNAs in children with idiopathic nephrotic syndrome. EBioMedicine 2019;39:552-561.

66. Alvarez S, Suazo C, Boltansky A, et al. Urinary exosomes as a source of kidney dysfunction biomarker in renal transplanta- 
tion. Transplant Proc 2013;45:3719-3723.

67. Zhang H, Huang E, Kahwaji J, et al. Plasma exosomes from HLA-sensitized kidney transplant recipients contain mRNA transcripts which predict development of antibody-mediated rejection. Transplantation 2017;101:2419-2428.

68. Park J, Lin HY, Assaker JP, et al. Integrated kidney exosome analysis for the detection of kidney transplant rejection. ACS Nano 2017;11:11041-11046.

69. Lim JH, Lee CH, Kim KY, et al. Novel urinary exosomal biomarkers of acute T cell-mediated rejection in kidney transplant recipients: a cross-sectional study. PLoS One 2018;13:e0204204.

70. Keri KC, Regner KR, Dall AT, Park F. Urinary exosomal expression of activator of $\mathrm{G}$ protein signaling 3 in polycystic kidney disease. BMC Res Notes 2018;11:359.

71. Solé C, Cortés-Hernández J, Felip ML, Vidal M, Ordi-Ros J. miR$29 \mathrm{c}$ in urinary exosomes as predictor of early renal fibrosis in lupus nephritis. Nephrol Dial Transplant 2015;30:1488-1496.

72. Tangtanatakul P, Klinchanhom S, Sodsai P, et al. Down-regulation of let-7a and miR-21 in urine exosomes from lupus nephritis patients during disease flare. Asian Pac J Allergy Immunol 2019;37:189-197.

73. Li Y, Xu X, Tang X, et al. MicroRNA expression profile of urinary exosomes in Type IV lupus nephritis complicated by cellular crescent. J Biol Res (Thessalon) 2018;25:16.

74. Chun-Yan L, Zi-Yi Z, Tian-Lin Y, et al. Liquid biopsy biomarkers of renal interstitial fibrosis based on urinary exosome. Exp Mol Pathol 2018;105:223-228.

75. Lange T, Artelt N, Kindt F, et al. MiR-21 is up-regulated in urinary exosomes of chronic kidney disease patients and after glomerular injury. J Cell Mol Med 2019;23:4839-4843.

76. Khurana R, Ranches G, Schafferer S, et al. Identification of urinary exosomal noncoding RNAs as novel biomarkers in chronic kidney disease. RNA 2017;23:142-152.

77. Dias F, Teixeira AL, Nogueira I, et al. Extracellular vesicles enriched in hsa-miR-301a-3p and hsa-miR-1293 dynamics in clear cell renal cell carcinoma patients: potential biomarkers of metastatic disease. Cancers (Basel) 2020;12:1450.

78. Dias F, Teixeira AL, Nogueira I, et al. Plasma extracellular vesicle-derived TIMP-1 mRNA as a prognostic biomarker in clear cell renal cell carcinoma: a pilot study. Int J Mol Sci 2020;21:4624.

79. Jiang XC, Gao JQ. Exosomes as novel bio-carriers for gene and drug delivery. Int J Pharm 2017;521:167-175.

80. Marcus ME, Leonard JN. FedExosomes: engineering therapeutic biological nanoparticles that truly deliver. Pharmaceuticals (Basel) 2013;6:659-680.
81. Kato M. Noncoding RNAs as therapeutic targets in early stage diabetic kidney disease. Kidney Res Clin Pract 2018;37:197-209.

82. Tang TT, Lv LL, Wang B, et al. Employing macrophage-derived microvesicle for kidney-targeted delivery of dexamethasone: an efficient therapeutic strategy against renal inflammation and fibrosis. Theranostics 2019;9:4740-4755.

83. Kalinec GM, Gao L, Cohn W, Whitelegge JP, Faull KF, Kalinec F. Extracellular vesicles from auditory cells as nanocarriers for anti-inflammatory drugs and pro-resolving mediators. Front Cell Neurosci 2019;13:530.

84. Yim N, Ryu SW, Choi K, et al. Exosome engineering for efficient intracellular delivery of soluble proteins using optically reversible protein-protein interaction module. Nat Commun 2016;7:12277.

85. Choi H, Kim Y, Mirzaaghasi A, et al. Exosome-based delivery of super-repressor Ik $\mathrm{B} \alpha$ relieves sepsis-associated organ damage and mortality. Sci Adv 2020;6:eaaz6980.

86. Gatti S, Bruno S, Deregibus MC, et al. Microvesicles derived from human adult mesenchymal stem cells protect against ischaemia-reperfusion-induced acute and chronic kidney injury. Nephrol Dial Transplant 2011;26:1474-1483.

87. Choi HY, Moon SJ, Ratliff BB, et al. Microparticles from kidney-derived mesenchymal stem cells act as carriers of proangiogenic signals and contribute to recovery from acute kidney injury. PLoS One 2014;9:e87853.

88. He J, Wang Y, Lu X, et al. Micro-vesicles derived from bone marrow stem cells protect the kidney both in vivo and in vitro by microRNA-dependent repairing. Nephrology (Carlton) 2015;20:591-600.

89. Choi HY, Lee HG, Kim BS, et al. Mesenchymal stem cell-derived microparticles ameliorate peritubular capillary rarefaction via inhibition of endothelial-mesenchymal transition and decrease tubulointerstitial fibrosis in unilateral ureteral obstruction. Stem Cell Res Ther 2015;6:18.

90. Wang B, Yao K, Huuskes BM, et al. Mesenchymal stem cells deliver exogenous microRNA-let7c via exosomes to attenuate renal fibrosis. Mol Ther 2016;24:1290-1301.

91. Nagaishi K, Mizue Y, Chikenji T, et al. Mesenchymal stem cell therapy ameliorates diabetic nephropathy via the paracrine effect of renal trophic factors including exosomes. Sci Rep 2016;6:34842.

92. Jiang ZZ, Liu YM, Niu X, et al. Exosomes secreted by human urine-derived stem cells could prevent kidney complications from type I diabetes in rats. Stem Cell Res Ther 2016;7:24.

93. Pan T, Jia P, Chen N, et al. Delayed remote ischemic preconditioning confersrenoprotection against septic acute kidney injury via exosomal miR-21. Theranostics 2019;9:405-423. 
94. Dominguez JH, Liu Y, Gao H, et al. Renal tubular cell-derived extracellular vesicles accelerate the recovery of established renal ischemia reperfusion injury. J Am Soc Nephrol 2017;28:35333544 .

95. Dominguez JM 2nd, Dominguez JH, Xie D, Kelly KJ. Human extracellular microvesicles from renal tubules reverse kidney ischemia-reperfusion injury in rats. PLoS One 2018;13:e0202550.

96. Yuana Y, Levels J, Grootemaat A, Sturk A, Nieuwland R. Co-isolation of extracellular vesicles and high-density lipoproteins using density gradient ultracentrifugation. J Extracell Vesicles 2014;3:10.3402/jev.v3.23262.
97. Baranyai T, Herczeg K, Onódi Z, et al. Isolation of exosomes from blood plasma: qualitative and quantitative comparison of ultracentrifugation and size exclusion chromatography methods. PLoS One 2015;10:e0145686.

98. Yang B, Chen Y, Shi J. Exosome biochemistry and advanced nanotechnology for next-generation theranostic platforms. Adv Mater 2019;31:e1802896.

99. Yang Z, Shi J, Xie J, et al. Large-scale generation of functional mRNA-encapsulating exosomes via cellular nanoporation. Nat Biomed Eng 2020;4:69-83.

100. Mossberg M, Ståhl AL, Kahn R, et al. C1-inhibitor decreases the release of vasculitis-like chemotactic endothelial microvesicles. JAm Soc Nephrol 2017;28:2472-2481. 